MATHEMATICS OF COMPUTATION

Volume 71, Number 240, Pages 1735-1743

S 0025-5718(01)01367-9

Article electronically published on November 21, 2001

\title{
COMPUTATION OF CLASS NUMBERS OF QUADRATIC NUMBER FIELDS
}

\author{
STÉPHANE LOUBOUTIN
}

\begin{abstract}
We explain how one can dispense with the numerical computation of approximations to the transcendental integral functions involved when computing class numbers of quadratic number fields. We therefore end up with a simpler and faster method for computing class numbers of quadratic number fields. We also explain how to end up with a simpler and faster method for computing relative class numbers of imaginary abelian number fields.
\end{abstract}

\section{INTRODUCTION}

Currently, the best available rigorous methods for computing class numbers of quadratic number fields $\mathbf{k}$ of discriminants $d_{\mathbf{k}}$ are of complexity $O\left(\left|d_{\mathbf{k}}\right|^{0.5+\epsilon}\right)$. However, assuming suitable forms of the generalized Riemann hypothesis, one can devise conditional but more efficient methods of lower complexity (see MoWi where a conditional method of complexity $O\left(d_{\mathbf{k}}^{0.2+\epsilon}\right)$ for computing class numbers of real quadratic fields is developed, and see [Coh Sections 5.5 and 5.9] where the conditional sub-exponential methods of McCurley and J. Buchmann for computing class groups of quadratic fields are developed). These rigorous methods stem from the analytic class number formulae (see Coh, Sections 5.3.3 and 5.6.2], [MoWi], [ScWa and $[\mathrm{WiBr}]$ ) and require the computation to sufficient accuracy of the transcendental integral functions $E(z)=\int_{z}^{\infty} e^{-x} x^{-1} d x$ (the exponential integral function) and $\operatorname{erfc}(z)=\frac{2}{\sqrt{\pi}} \int_{z}^{\infty} e^{-x^{2}} d x$ (the complementary error function) by using the following power series expansions (if $z$ is small) and continued fractional expansions (if $z$ is large):

$$
\begin{aligned}
\int_{z}^{\infty} e^{-x^{2}} d x & =\frac{1}{2} \sqrt{\pi}-\sum_{n \geq 0} \frac{(-1)^{n} z^{2 n+1}}{n !(2 n+1)} \\
& =\frac{1}{2} e^{-z^{2}}\left(\frac{1}{z+} \frac{\frac{1}{2}}{z+} \frac{1}{z+} \frac{\frac{3}{2}}{z+} \frac{2}{z+} \frac{\frac{5}{2}}{z+} \cdots\right), \\
\int_{z}^{\infty} e^{-x} \frac{d x}{x} & =-\gamma-\log (z)-\sum_{n \geq 1} \frac{(-1)^{n} z^{n}}{n \cdot n !} \\
& =e^{-z}\left(\frac{1}{z+} \frac{1}{1+} \frac{1}{z+} \frac{2}{1+} \frac{2}{z+} \frac{3}{1+} \frac{3}{z+} \cdots\right)
\end{aligned}
$$

Received by the editor March 29, 2000 and, in revised form, November 27, 2000.

2000 Mathematics Subject Classification. Primary 11R11, 11R29, 11R21, 11Y35.

Key words and phrases. Quadratic number field, class number, Dirichlet $L$-function, relative class number. 
(here $\gamma=0.577 \cdots$ denotes Euler's constant). In this paper we explain how one can dispense with these evaluations, thus greatly simplifying the implementation of these rigorous methods for computing class numbers of quadratic number fields, and making them faster but still of the same complexity $O\left(\left|d_{\mathbf{k}}\right|^{0.5+\epsilon}\right)$. In contrast with what is usually done, we will write all our class number formulae at $s=0$. Indeed, values at $s=0$ of $L$-functions associated with odd Dirichlet characters are algebraic numbers, and we explained in [Lou3] how useful this observation is for computing their exact values from the computation of their numerical approximations (see also [Lou5] where we use [Lou4 to generalize the method developed in Lou3 for computing relative class numbers of nonabelian CM-fields).

Let us now set some of the notation we will be using throughout this paper. We let $\chi$ be a primitive Dirichlet character modulo $f>1$. We set

$$
S_{n}(\chi)=\sum_{k=1}^{n} \chi(k) \text { and } T_{n}(\chi)=\sum_{k=1}^{n} \frac{1}{k} \chi(k) .
$$

We also set $\alpha=\sqrt{\pi / f}, e_{n}=e^{-\pi n^{2} / f}=e^{-n^{2} \alpha^{2}}$ and

$$
\tau(\chi)=\sum_{x=1}^{f-1} \chi(x) e^{2 x \pi i / f} .
$$

For $t>0$ real and $M \geq 0$ real, we set

$$
B(t, M, f)=\sqrt{f(t \log (f / \pi)+M) / \pi}=\alpha^{-1} \sqrt{M-2 t \log \alpha}
$$

and assume $f \neq 3$, which implies $f>\pi, 0<\alpha<1, B(t, M, f) \geq \sqrt{f / \pi}$ and $e^{-m^{2} \alpha^{2}} \leq \alpha^{2 t} e^{-M}$ for $m \geq B(t, M, f)$. Finally, we will use:

Lemma 1. Let $\alpha>0$ be real and $g$ of class $\mathcal{C}^{2}$ in the range $) 0,+\infty$ ( be given. Then for any positive rational integer $n \geq 1$ we have

$$
\int_{n \alpha}^{(n+1) \alpha} g=\alpha \frac{g((n+1) \alpha)+g(n \alpha)}{2}+\frac{\theta \alpha^{2}}{8} \int_{n \alpha}^{(n+1) \alpha}\left|g^{\prime \prime}\right| \quad(|\theta| \leq 1) .
$$

Proof. Set $B_{2}(x)=x(x-1) / 2$. Then the reader will check that we have

$$
\int_{n \alpha}^{(n+1) \alpha} g(x) d x=\alpha \frac{g((n+1) \alpha)+g(n \alpha)}{2}+\alpha^{3} \int_{0}^{1} B_{2}(x) g^{\prime \prime}(\alpha(x+n)) d x .
$$

Now, the bound $0 \leq\left|B_{2}(x)\right| \leq 1 / 8$ for $0 \leq u \leq 1$ yields the desired result.

\section{IMAGINARY ABELIAN NUMBER FIELDS}

Let $\chi$ be a primitive odd Dirichlet character modulo $f>3$. Set $W(\chi)=$ $i^{-1} \tau(\chi) / \sqrt{f}$, which has absolute value equal to one. We can express $L(0, \chi)$ as the limit of rapidly absolutely convergent series (see [Dav] or Lou3])

$$
L(0, \chi)=\frac{1}{\sqrt{\pi}}\left(\frac{W(\chi)}{\alpha} \sum_{n \geq 1} \frac{\bar{\chi}(n)}{n} e_{n}+2 \sum_{n \geq 1} \chi(n) \int_{n \alpha}^{\infty} e^{-x^{2}} d x\right)
$$

and

$$
L(0, \chi)=\frac{1}{\sqrt{\pi}}\left(\frac{W(\chi)}{\alpha} \sum_{n \geq 1} \frac{\bar{\chi}(n)}{n} e_{n}+2 \sum_{n \geq 1} S_{n}(\chi) \int_{n \alpha}^{(n+1) \alpha} e^{-x^{2}} d x\right) .
$$


Proposition 2 (Compare with Proposition [7). Let $\chi$ be a primitive odd Dirichlet character of conductor $f$. For some $\theta$ satisfying $|\theta| \leq 1$, it holds that

$$
L(0, \chi)=\frac{1}{\sqrt{\pi}}\left(\frac{W(\chi)}{\alpha} \sum_{n \geq 1} \frac{\bar{\chi}(n)}{n} e_{n}+\alpha \sum_{n \geq 1}\left(e_{n}+e_{n+1}\right) S_{n}(\chi)\right)+\frac{3 \theta}{8 f^{1 / 2}} .
$$

Proof. Applying Lemma 1 to $g(x)=e^{-x^{2}}$ and noticing that $\left|S_{n}(\chi)\right| \leq n$, we obtain

$$
2 \sum_{n \geq 1} S_{n}(\chi) \int_{n \alpha}^{(n+1) \alpha} e^{-x^{2}} d x=\alpha \sum_{n \geq 1}\left(e_{n}+e_{n+1}\right) S_{n}(\chi)+\frac{\theta \alpha}{4} R^{\prime}
$$

with $|\theta| \leq 1$ and

$$
\begin{aligned}
R^{\prime} & =\alpha \sum_{n \geq 1} n \int_{n \alpha}^{(n+1) \alpha}\left|g^{\prime \prime}\right| \\
& =\alpha \sum_{n \geq 1} \int_{n \alpha}^{\infty}\left|g^{\prime \prime}\right| \\
& \leq \alpha \int_{0}^{\infty}\left(\int_{\alpha u}^{\infty}\left|g^{\prime \prime}(x)\right| d x\right) d u=\int_{0}^{\infty} x\left|g^{\prime \prime}(x)\right| d x \stackrel{\text { def }}{=} R_{o d d}
\end{aligned}
$$

which, for $g(x)=e^{-x^{2}}$, yields

$$
R_{o d d}=\int_{0}^{\infty} x\left|4 x^{2}-2\right| e^{-x^{2}} d x=(4 / \sqrt{e})-1=1.426 \cdots \leq 3 / 2 .
$$

Using (7), (8) and (9), we obtain the desired result.

Proposition 3. Assume $f>3, t>0$ and $M \geq 1$. For any positive rational integer $m \geq B(t, M, f)$ it holds that

$$
\begin{aligned}
\text { and } \quad & \left|\frac{W(\chi)}{\alpha} \sum_{n>m} \frac{\bar{\chi}(n)}{n} e_{n}\right| \leq \frac{1}{\alpha} \sum_{n>m} \frac{1}{n} e_{n} \leq \frac{1}{2} \alpha^{2 t-1} e^{-M} \\
& \left|\alpha \sum_{n>m}\left(e_{n}+e_{n+1}\right) S_{n}(\chi)\right| \leq \alpha \sum_{n>m} 2 n e_{n} \leq \alpha^{2 t-1} e^{-M} .
\end{aligned}
$$

Proof. For $m \geq B(t, M, f)$ we have

$$
\begin{aligned}
& \frac{1}{\alpha} \sum_{n>m} \frac{1}{n} e^{-n^{2} \alpha^{2}} \leq \frac{1}{\alpha} \int_{m}^{\infty} x e^{-x^{2} \alpha^{2}} \frac{d x}{x^{2}} \\
& \quad \leq \frac{1}{\alpha m^{2}} \int_{m}^{\infty} x e^{-x^{2} \alpha^{2}} d x=\frac{e^{-m^{2} \alpha^{2}}}{2 m^{2} \alpha^{3}} \leq \frac{\alpha^{2 t-1} e^{-M}}{2(M-2 t \log \alpha)} \leq \alpha^{2 t-1} e^{-M} / 2
\end{aligned}
$$

and

$$
\alpha \sum_{n>m} 2 n e^{-n^{2} \alpha^{2}} \leq 2 \alpha \int_{m}^{\infty} x e^{-m^{2} x^{2}} d x=\frac{1}{\alpha} e^{-m^{2} \alpha^{2}} \leq \alpha^{2 t-1} e^{-M}
$$

According to Propositions 2 and 3 , we obtain 
Theorem 4 (Compare with Theorem 9 below). Let $M \geq 1$ be given, let $\chi$ be a primitive odd Dirichlet character of conductor $f$, let $m$ be the least rational integer greater than or equal to $B\left(\frac{1}{2}, M, f\right)=O\left(f^{0.5+\epsilon}\right)$ and set

$$
L_{M}(0, \chi)=\frac{1}{\sqrt{\pi}}\left(\frac{W(\chi)}{\alpha} \sum_{n=1}^{m} \frac{\bar{\chi}(n)}{n} e_{n}+\alpha \sum_{n=1}^{m}\left(e_{n}+e_{n+1}\right) S_{n}(\chi)\right),
$$

where $\alpha=\sqrt{\pi / f}, e_{n}=e^{-n^{2} \alpha^{2}}$ and $S_{n}(\chi)$ is defined in (5). Then,

$$
\left|L(0, \chi)-L_{M}(0, \chi)\right| \leq \frac{3}{2 \sqrt{\pi}} e^{-M}+\frac{3}{8 \sqrt{f}} .
$$

Remark 5.

1. Of particular importance is the case where $\chi$ is the primitive quadratic odd Dirichlet character of conductor $f=\left|d_{\mathbf{k}}\right|$ associated with an imaginary quadratic field $\mathbf{k}$ of discriminant $d_{\mathbf{k}}<-4$ and class number $h_{\mathbf{k}}$. Then, $\bar{\chi}=\chi$, $W(\chi)=+1, h_{\mathbf{k}}=L(0, \chi)$ and Theorem 4 provides us with a much more satisfactory result than [Lou1, Theorem 1].

2. In practice, we do not compute all the $e_{n}=\exp \left(-\pi n^{2} / f\right)$ 's for $1 \leq n \leq m$ by using the exponential function. It is more efficient to compute the $e_{n}$ 's inductively by setting $f_{n}=\exp (-\pi(2 n+1) / f)$, by computing $f_{0}=\exp (-\pi / f)$ and $h=\exp (-2 \pi / f)$ and by using the induction formulae $f_{n+1}=h f_{n}$ and $e_{n+1}=e_{n} f_{n}$. In this process, at each step $n$, instead of performing the computation of $\exp \left(-\pi n^{2} / f\right)$ we only perform two multiplications.

3. We explained in [Lou3] how to compute relative class numbers of imaginary abelian number fields of a given degree by computing numerical approximations to linear combinations with bounded coefficients of values at $s=0$ of $L$-functions associated with odd primitive Dirichlet characters. Therefore, combining Theorem 4 and the method developed in Lou3], we end up with an efficient method for computing relative class numbers of imaginary abelian number fields of a given degree. This method does not require us to compute approximations to transcendental integral functions.

4. We also explained in [Lou5] how to compute relative class numbers of CMfields by computing numerical approximations to linear combinations with bounded coefficients of values at $s=0$ of Heckes's $L$-functions associated with characters on strict ray class groups.

Therefore, in order to extend our present method further, we would like to find a method (generalizing Proposition 2 and Proposition 3) which would enable us to dispense with the computation of numerical approximations to the complicated integral transcendental functions involved when computing numerical approximations to values at $s=0$ of such Hecke's $L$-functions (see [Lou2] and [Lou4]).

\section{ReAL QUADRATIC NUMBER FIELDS}

Let $\chi$ be a primitive even Dirichlet character modulo $f>3$. Set $W(\chi)=$ $\tau(\chi) / \sqrt{f}$, which has absolute value equal to one. Then $L(0, \chi)=0$ and we can express the derivative $L^{\prime}(0, \chi)$ as the limit of rapidly absolutely convergent series (use Dav]):

$$
L^{\prime}(0, \chi)=\sum_{n \geq 1} \chi(n) \int_{n \alpha}^{\infty} e^{-x^{2}} \frac{d x}{x}+\frac{W(\chi)}{\alpha} \sum_{n \geq 1} \frac{\bar{\chi}(n)}{n} \int_{n \alpha}^{\infty} e^{-x^{2}} d x
$$


and

$$
L^{\prime}(0, \chi)=\sum_{n \geq 1} S_{n}(\chi) \int_{n \alpha}^{(n+1) \alpha} e^{-x^{2}} \frac{d x}{x}+\frac{W(\chi)}{\alpha} \sum_{n \geq 1} T_{n}(\bar{\chi}) \int_{n \alpha}^{(n+1) \alpha} e^{-x^{2}} d x .
$$

Lemma 6. For $n \geq 1$, set

$$
u_{n}=\frac{e_{n}}{n}+\frac{e_{n+1}}{n+1}+2 \log \left(\frac{n+1}{n}\right)-\frac{1}{n}-\frac{1}{n+1} .
$$

For any $m \geq 1$ we have

$$
\sum_{n \geq 1} S_{n}(\chi) \int_{n \alpha}^{(n+1) \alpha} e^{-x^{2}} \frac{d x}{x}=\frac{1}{2} \sum_{n \geq 1} u_{n} S_{n}(\chi)+\frac{\theta \alpha}{4}
$$

for some $\theta$ satisfying $|\theta| \leq 1$, and

$$
\frac{1}{\alpha} \sum_{n \geq 1} T_{n}(\bar{\chi}) \int_{n \alpha}^{(n+1) \alpha} e^{-x^{2}} d x=\frac{1}{2} \sum_{n \geq 1}\left(e_{n}+e_{n+1}\right) T_{n}(\bar{\chi})+\frac{\theta \alpha}{4} \log (e / \alpha)
$$

for some $\theta$ satisfying $|\theta| \leq 1$.

Proof. Set $g(x)=\left(e^{-x^{2}}-1\right) / x$. Then $x g^{\prime \prime}(x)=\left(4 x^{2}+2\right) e^{-x^{2}}+2\left(e^{-x^{2}}-1\right) / x^{2}$ and according to Lemma 1 we obtain

$$
\begin{aligned}
& \sum_{n=1}^{m} S_{n}(\chi) \int_{n \alpha}^{(n+1) \alpha} e^{-x^{2}} \frac{d x}{x} \\
& \quad=\sum_{n=1}^{m} S_{n}(\chi) \int_{n \alpha}^{(n+1) \alpha} g(x) d x+\sum_{n=1}^{m} S_{n}(\chi) \log \left(\frac{n+1}{n}\right) \\
& \quad=\alpha \sum_{n=1}^{m} \frac{g((n+1) \alpha)+g(n \alpha)}{2} S_{n}(\chi)+\sum_{n=1}^{m} S_{n}(\chi) \log \left(\frac{n+1}{n}\right)+\frac{\theta \alpha}{8} R^{\prime \prime} \\
& \quad=\frac{1}{2} \sum_{n=1}^{m} u_{n} S_{n}(\chi)+\frac{\theta \alpha}{8} R^{\prime \prime}
\end{aligned}
$$

where, as in the proof of Proposition 2, we have

$$
R^{\prime \prime}=\alpha \sum_{n=1}^{m} n \int_{n \alpha}^{(n+1) \alpha}\left|g^{\prime \prime}\right| \leq \int_{0}^{\infty} x\left|g^{\prime \prime}(x)\right| d x \stackrel{\text { def }}{=} R_{\text {even }}=2\left(\beta g^{\prime}(\beta)-g(\beta)\right)
$$

where $\beta=1.792641 \cdots$ is the only positive real zero of $g^{\prime \prime}$. Hence, $R^{\prime \prime} \leq R_{\text {even }}=$ $4\left(1-\left(1+\beta^{2}\right) e^{-\beta^{2}}\right) / \beta=1.853264 \cdots \leq 2$.

Applying Lemma 1 to $g(x)=e^{-x^{2}}$, we obtain

$$
\frac{1}{\alpha} \sum_{n \geq 1} T_{n}(\bar{\chi}) \int_{n \alpha}^{(n+1) \alpha} e^{-x^{2}} d x=\frac{1}{2} \sum_{n \geq 1}\left(e_{n}+e_{n+1}\right) T_{n}(\bar{\chi})+\frac{\theta \alpha}{8} R^{\prime \prime \prime}
$$


with $|\theta| \leq 1$ and

$$
\begin{aligned}
R^{\prime \prime \prime} & =\sum_{n \geq 1}\left(\sum_{k=1}^{n} \frac{1}{k}\right) \int_{n \alpha}^{(n+1) \alpha}\left|g^{\prime \prime}\right| \\
& =\sum_{n \geq 1} \frac{1}{n} \int_{n \alpha}^{\infty}\left|g^{\prime \prime}\right| \\
& \leq \int_{\alpha}^{\infty}\left|g^{\prime \prime}(x)\right| d x+\int_{1}^{\infty}\left(\int_{\alpha u}^{\infty}\left|g^{\prime \prime}(x)\right| d x\right) \frac{d u}{u} \\
& =\int_{\alpha}^{\infty}\left|g^{\prime \prime}(x)\right| d x+\int_{\alpha}^{\infty}\left|g^{\prime \prime}(x)\right| \log (x / \alpha) d x \\
& \leq \int_{0}^{\infty}\left|g^{\prime \prime}(x)\right| \log (e x / \alpha) d x \\
& =\frac{4}{\sqrt{2 e}} \log (e / \alpha \sqrt{2})-2 \int_{0}^{1 / \sqrt{2}} e^{-x^{2}} d x+2 \int_{1 / \sqrt{2}}^{\infty} e^{-x^{2}} d x \\
& \leq \frac{1}{2}-\frac{7}{4} \log \alpha \leq 2 \log (e / \alpha),
\end{aligned}
$$

and using (16), we obtain the desired result.

According to (12) and to Lemma 6 (where we take the limit as $m$ goes to infinity in (14)), we obtain

Proposition 7 (Compare with Proposition 2). Let $\chi$ be a primitive even Dirichlet character of conductor $f>1$. For some $\theta$ satisfying $|\theta| \leq 1$, it holds that

$$
L^{\prime}(0, \chi)=\frac{1}{2} \sum_{n \geq 1} u_{n} S_{n}(\chi)+\frac{W(\chi)}{2} \sum_{n \geq 1}\left(e_{n}+e_{n+1}\right) T_{n}(\bar{\chi})+\frac{\theta \alpha}{4} \log \left(e^{2} / \alpha\right) .
$$

Proposition 8. Assume $f>3, t>0$ and $M \geq 1$. For any positive rational integer $m \geq B(t, M, f)$ it holds that

$$
\left|\sum_{n>m} S_{n}(\chi) \int_{n \alpha}^{(n+1) \alpha} e^{-x^{2}} \frac{d x}{x}\right| \leq \sum_{n>m} e_{n} \leq \frac{1}{2} \alpha^{2 t-1} e^{-M}
$$

and

$$
\left|\frac{1}{2} \sum_{n>m}\left(e_{n}+e_{n+1}\right) T_{n}(\bar{\chi})\right| \leq \frac{1}{2} \alpha^{2 t-1} e^{-M} \log (e / \alpha) .
$$

Proof. We have

$$
\begin{aligned}
& \left|\sum_{n>m} S_{n}(\chi) \int_{n \alpha}^{(n+1) \alpha} e^{-x^{2}} \frac{d x}{x}\right| \leq \sum_{n>m} n \int_{n \alpha}^{(n+1) \alpha} e^{-x^{2}} \frac{d x}{x} \\
& \quad \leq \sum_{n>m} e^{-n^{2} \alpha^{2}} \leq \frac{1}{m} \int_{m}^{\infty} x e^{-x^{2} \alpha^{2}} d x=\frac{e^{-m^{2} \alpha^{2}}}{2 m \alpha^{2}} \leq \frac{\alpha^{2 t-1} e^{-M}}{2 \sqrt{M-2 t \log \alpha}} .
\end{aligned}
$$

In the same way, we have

$$
R_{m} \stackrel{\text { def }}{=}\left|\frac{1}{2} \sum_{n>m}\left(e_{n}+e_{n+1}\right) T_{n}(\bar{\chi})\right| \leq \sum_{n>m}\left(\sum_{k=1}^{n} \frac{1}{k}\right) e_{n} \leq \sum_{n>m} \log (e n) e^{-n^{2} \alpha^{2}} .
$$


Since $M \geq 1$ and $t>0$ imply $m \geq B(t, M, f) \geq 1 / \alpha$ and since $x \mapsto \log (e x) e^{-\alpha^{2} x^{2}}$ decreases in the range $x \geq 1 / \alpha$, we obtain

$$
R_{m} \leq \int_{m}^{\infty} \frac{\log (e x)}{x} x e^{-\alpha^{2} x^{2}} d x \leq \frac{\log (e m) e^{-m^{2} \alpha^{2}}}{2 m \alpha^{2}} \leq \frac{1}{2} \alpha^{2 t-1} e^{-M} \frac{\log (e u / \alpha)}{u}
$$

where $u=\sqrt{M-2 t \log \alpha} \geq 1$. Since $u \mapsto \frac{\log (e u / \alpha)}{u}$ decreases in the range $u \geq \alpha$ and since we have $u \geq 1>\alpha$, we obtain the desired result.

According to (12), (14), (15), (17) and (18), we obtain

Theorem 9 (Compare with Theorem 4). Let $M \geq 1$ be given, let $\chi$ be the primitive even Dirichlet character of conductor $f>1$, let $m$ be the least rational integer geater than or equal to $B\left(\frac{1}{2}, M, f\right)=O\left(f^{0.5+\epsilon}\right)$ and set

$$
L_{M}^{\prime}(0, \chi)=\frac{1}{2} \sum_{n=1}^{m} u_{n} S_{n}(\chi)+\frac{1}{2} \sum_{n=1}^{m}\left(e_{n}+e_{n+1}\right) T_{n}(\bar{\chi}),
$$

where $\alpha=\sqrt{\pi / f}, e_{n}=e^{-n^{2} \alpha^{2}}, S_{n}(\chi)$ and $T_{n}(\chi)$ are defined in (15) and $u_{n}$ is defined in (13). Then,

$$
\left|L^{\prime}(0, \chi)-L_{M}^{\prime}(0, \chi)\right| \leq \frac{\alpha+2 e^{-M}}{4} \log \left(e^{2} / \alpha\right)
$$

Remark 10.

1. Of particular importance is the case where $\chi$ is the primitive quadratic even Dirichlet character of conductor $f=d_{\mathbf{k}}$ associated with a real quadratic field $\mathbf{k}$ of discriminant $d_{\mathbf{k}}$, fundamental unit $\epsilon_{\mathbf{k}}>1$, and class number $h_{\mathbf{k}}$. Then, $\bar{\chi}=\chi, W(\chi)=+1, h_{\mathbf{k}}=L^{\prime}(0, \chi) / \log \epsilon_{\mathbf{k}}$ and Theorem 9 yields

$$
\left|h_{\mathbf{k}}-L_{M}^{\prime}(0, \chi) / \log \epsilon_{\mathbf{k}}\right| \leq \frac{5}{4} \sqrt{\frac{\pi}{d_{\mathbf{k}}}}+\frac{5}{2} e^{-M}
$$

(for $\epsilon_{\mathbf{k}} \geq\left(\sqrt{d_{\mathbf{k}}-4}+\sqrt{d_{\mathbf{k}}}\right) / 2$ yields $\left.\log \left(e^{2} / \alpha\right) \leq 5 \log \epsilon_{\mathbf{k}}\right)$. We refer the reader to [WiBr Section 2] for the evaluation of the regulator $\log \epsilon_{\mathbf{k}}$ of a real quadratic field $\mathbf{k}$ by using an elementary algorithm of complexity $O\left(d_{\mathbf{k}}^{0.5+\epsilon}\right)$ based on the use of continued fractional expansions.

2. Here again, the second point of Remark $[5$ applies.

3. In contrast with Theorem 4 (see Point 2 of Remark 5), Theorem 9 cannot be used to compute class numbers of nonquadratic real abelian numbers fields of a given degree (for it is not known how to reduce their computation to the computation of numerical approximations to linear combinations with bounded coefficients of values at $s=0$ of derivatives of $L$-functions associated with even characters (compare with [Lou3])).

\section{NumericAL EXAMPLES}

To assess the efficiency of the present technique for computing class numbers $h_{\mathbf{k}}$ of quadratic number fields $\mathbf{k}$ of large discriminants $d_{\mathbf{k}}$, we programmed our formulas (10) and (19) with $M=3$ in Kida's language UBASIC, which allows fast arbitrary precision calculation on PC's (the precision of real numbers in significant digits we used was equal to 28). Let us detail how much our method improves upon the previous ones based on the use of (6) and Point 1 of Remark 5 for $d_{\mathbf{k}}<0$, and of (11) and Point 1 of Remark 10 for $d_{\mathbf{k}}>0$ (see [Coh] and [WiBr]). We give all the 
TABLE 1 . The imaginary quadratic case

\begin{tabular}{|rrrrrrr|}
\hline$d_{\mathbf{k}}<0$ & $T_{1}$ & $T_{2}$ & $T_{3}$ & $T_{4}$ & $h_{\mathbf{k}}$ \\
\hline $5-10^{10}$ & 4 & 10 & 31 & 24 & 38272 \\
$5-10^{11}$ & 12 & 34 & 100 & 78 & 95840 \\
$5-10^{12}$ & 41 & 112 & 323 & 251 & 506880 \\
$5-10^{13}$ & 138 & 376 & 1053 & 812 & 1051452 \\
$5-10^{14}$ & 460 & 1224 & 3391 & 2619 & 3312448 \\
\hline
\end{tabular}

details only in the case that $\mathbf{k}$ is imaginary. Set $\alpha_{\mathbf{k}}=\sqrt{\pi /\left|d_{\mathbf{k}}\right|}, e_{n}=\exp \left(-n^{2} \alpha_{\mathbf{k}}^{2}\right)$, $m_{\mathbf{k}}=B\left(\frac{1}{2}, M,\left|d_{\mathbf{k}}\right|\right)=\sqrt{\left|d_{\mathbf{k}}\right|\left(\log \left(\left|d_{\mathbf{k}}\right| / \pi\right)+2 M\right) / 2 \pi}$,

$$
h_{\mathbf{k}}(M)=\frac{1}{\sqrt{\pi}}\left(\frac{1}{\alpha_{\mathbf{k}}} \sum_{n=1}^{m_{\mathbf{k}}} \frac{\chi_{\mathbf{k}}(n)}{n} e_{n}+\alpha_{\mathbf{k}} \sum_{n=1}^{m_{\mathbf{k}}}\left(e_{n}+e_{n+1}\right) S_{n}\left(\chi_{\mathbf{k}}\right)\right)
$$

(see Theorem 4 and Point 1 of Remark 5 ) for which

$$
R_{\mathbf{k}}(M):=\left|h_{\mathbf{k}}-h_{\mathbf{k}}(M)\right| \leq \frac{3}{2 \sqrt{\pi}} e^{-M}+\frac{3}{8 \sqrt{\left|d_{\mathbf{k}}\right|}},
$$

and

$$
h_{\mathbf{k}}^{\prime}(M)=\frac{1}{\sqrt{\pi}}\left(\frac{1}{\alpha_{\mathbf{k}}} \sum_{n=1}^{m_{\mathbf{k}}} \frac{\chi_{\mathbf{k}}(n)}{n} e_{n}+2 \sum_{n=1}^{m_{\mathbf{k}}} \chi_{\mathbf{k}}(n) \int_{n \alpha_{\mathbf{k}}}^{\infty} e^{-x^{2}} d x\right)
$$

(see (6) and Point 1 of Remark (5) for which

$$
R_{\mathbf{k}}^{\prime}(M):=\left|h_{\mathbf{k}}-h_{\mathbf{k}}^{\prime}(M)\right| \leq \frac{1}{\sqrt{\pi}} e^{-M}
$$

(notice that $2 \int_{X}^{\infty} e^{-x^{2}} d x \leq \frac{1}{X} \int_{X}^{\infty} 2 x e^{-x^{2}} d x=e^{-X^{2}} / X$ and use Proposition 3 with $t=0.5)$. Notice that the number of terms in the truncated sums (20) and (22) are equal, and that the error terms $R_{\mathrm{k}}$ and $R_{\mathrm{k}}^{\prime}$ are of the same quality. Now, the previously known rigorous method for computing $h_{\mathbf{k}}$ consists in using (22) and the power series expansion (11) for small values of $z$ and continued fraction expansion (2) for large values of $z$ to compute approximations to $h_{\mathbf{k}}^{\prime}$. The main drawbacks of this method are (i) that we must carefully explain how many terms we have to consider in (11) and (2) to end up with good enough approximations to each $\operatorname{erfc}\left(n \alpha_{\mathbf{k}}\right)$, (ii) that computing $\operatorname{erfc}\left(n \alpha_{\mathbf{k}}\right)$ is slower than computing $e_{n}=\exp \left(-n^{2} \alpha_{\mathbf{k}}^{2}\right)$ and (iii) that we cannot take advantage of the second point of our Remark 5 while dealing with the indices for which we use (1) for computing approximations to $\operatorname{erfc}\left(n \alpha_{\mathbf{k}}\right)$. Instead, by using (20) and Point 2 of Remark 5 we do not meet with any of these drawbacks and end up with a faster and easier to implement method for computing $h_{\mathbf{k}}$.

We present in Tables 1 and 2 the results of applying these methods to compute class numbers of five imaginary quadratic fields with various size discriminants and of five real quadratic fields with various size discriminants and regulators. The computations were all carried out on a PC microcomputer with Pentium III, $333 \mathrm{Mhz}$. Here, $T_{1}$ is the time required to compute $h_{\mathbf{k}}$ when using (20) and the second point of Remark 5, $T_{2}$ is the time required to compute $h_{\mathbf{k}}$ when using (20) but when disregarding the second point of Remark $5, T_{3}$ is the time required to compute $h_{\mathbf{k}}$ when using (22), (11) for $n \alpha_{\mathbf{k}}=z<1.8$ and (2) for $n \alpha_{\mathbf{k}}=z \geq 1.8$ and, finally, $T_{4}$ is the time required to compute $h_{\mathbf{k}}$ when using (22), (11) for $n \alpha_{\mathbf{k}}=z<1.8$ and (2) 
TABLE 2. The real quadratic case

\begin{tabular}{|rrrrrrr|}
\hline$d_{\mathbf{k}}>0$ & $T_{0}$ & $T_{1}$ & $T_{2}$ & $T_{3}$ & $T_{4}$ & $h_{\mathbf{k}}$ \\
\hline $10^{10}+5$ & 0 & 8 & 15 & 51 & 41 & 1134 \\
$10^{11}+21$ & 4 & 27 & 49 & 167 & 132 & 2 \\
$10^{12}+1$ & 0 & 90 & 161 & 545 & 427 & 50280 \\
$10^{13}+1$ & 48 & 297 & 532 & 1763 & 1374 & 2 \\
$10^{14}+5$ & 0 & 959 & 1729 & 5706 & 4417 & 107920 \\
\hline
\end{tabular}

for $n \alpha_{\mathbf{k}}=z \geq 1.8$ and the second point of Remark 5 to compute the $\exp \left(-\left(n \alpha_{\mathbf{k}}\right)^{2}\right)^{\prime}$ 's in (2) for the $n$ 's for which $n \alpha_{\mathbf{k}}=z \geq 1.8$ All these $T_{i}$ are expressed in seconds. Here, $T_{0}$ denotes the time required to compute $\log \epsilon_{\mathbf{k}}$ by using continued fractions (see $[\mathrm{WiBr}]$ ), and $T_{1}, T_{2}, T_{3}$ and $T_{4}$ are as in Table 1 .

These Tables 1 and 2 clearly show that our method is significantly faster in practice than existing rigorous methods.

\section{REFERENCES}

[Coh] H. Cohen. A Course in Computational Algebraic Number Theory. Springer-Verlag, Grad. Texts Math. 138, 1993. MR 94i:11105

[Dav] H. Davenport. Multiplicative Number Theory, The functional Equation of the LFunctions. Springer-Verlag, Grad. Texts Math. 74 (1980), Chapter 9. MR 82m:10001

[Lou1] S. Louboutin. $L$-functions and class numbers of imaginary quadratic fields and of quadratic extensions of an imaginary quadratic field. Math. Comp. 59 (1992), 213-230. MR 92k:11128

[Lou2] S. Louboutin. Computation of relative class numbers of CM-fields. Math. Comp. 66 (1997), 173-184. MR 97k:11157

[Lou3] S. Louboutin. Computation of relative class numbers of imaginary abelian number fields. Experimental Math. 7 (1998), 293-303. MR 2000c:11207

[Lou4] S. Louboutin. Computation of relative class numbers of CM-fields by using Hecke $L$ functions. Math. Comp. 69 (2000), 371-393. MR 2000i:11172

[Lou5] S. Louboutin. Computation of $L(0, \chi)$ and of relative class numbers of CM-fields. Nagoya Math. J. 161 (2001), 171-191. CMP 2001:09

[MoWi] R. A. Mollin and H. C. Williams. Computation of the class number of a real quadratic field. Utilitas Math. 41 (1992), 259-308. MR 93d:11134

[ScWa] R. Schoof and L. C. Washington. Quintic polynomials and real cyclotomic fields with large class numbers. Math. Comp. 50 (1988), 543-556. MR 89h:11067b

[StWi] A. J. Stephens and H. C. Williams. Computation of real quadratic fields with class number one. Math. Comp. 51 (1988), 809-824. MR 90b:11106

[WiBr] H. C. Williams and J. Broere. A computational technique for evaluating $L(1, \chi)$ and the class number of a real quadratic field. Math. Comp. 30 (1976), 887-893. MR 54:2623]

Institut de Mathématiques de Luminy, UPR 906, 163, avenue de Luminy, Case 907, 13288 Marseille Cedex 9, France

E-mail address: loubouti@iml.univ-mrs.fr 